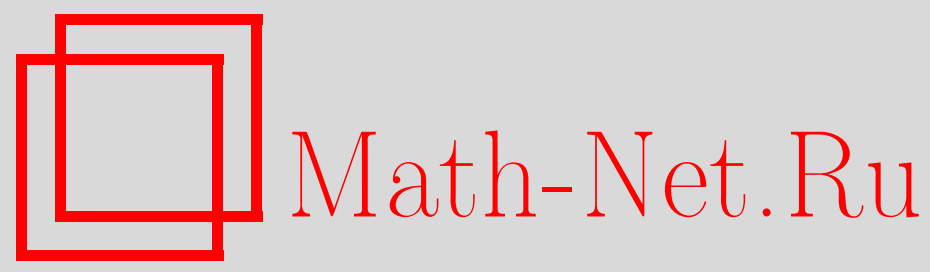

И. В. Тихонов, Ю. С. Эйдельман, Критерий единственности в обратной задаче для абстрактного дифференциального уравнения с нестационарным неоднородным слагаемым, Матем. заметки, 2005, том 77, выпуск 2, 273-290

DOI: https://doi.org/10.4213/mzm2489

Использование Общероссийского математического портала Math-Net.Ru подразумевает, что вы прочитали и согласны с пользовательским соглашением http://www . mathnet.ru/rus/agreement

Параметры загрузки:

IP : 3.85 .7 .115

26 апреля 2023 г., 14:14:59

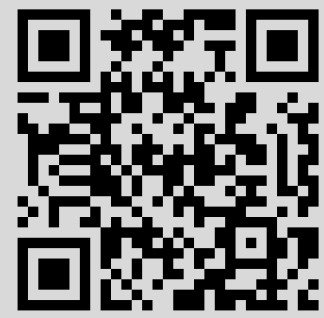




\title{
КРИТЕРИЙ ЕДИНСТВЕННОСТИ В ОБРАТНОЙ ЗАДАЧЕ ДЛЯ АБСТРАКТНОГО ДИФФЕРЕНЦИАЛЬНОГО УРАВНЕНИЯ С НЕСТАЦИОНАРНЫМ НЕОДНОРОДНЫМ СЛАГАЕМЫМ
}

\author{
И.В. Тихонов, Ю.С. Эйдельман
}

В банаховом пространстве $E$ рассматривается обратная задача $d u(t) / d t=A u(t)+$ $\varphi(t) p, u(0)=u_{0}, u(T)=u_{1}$, с неизвестной функцией $u(t)$ и элементом $p \in E$. Оператор $A$ предполагается линейным и замкнутым. В работе указаны минимальные ограничения на функцию $\varphi \in C([0, T])$, при которых единственность решения обратной задачи полностью выражается в терминах собственных значений оператора $A$.

Библиографория: 25 названий.

1. Предварительные рассмотрения. Пусть $E$-комплексноебанаховопространство. Пусть $A$ - линейньй замкнутьй оператор в $E$ с областью определения $D(A) \subset E$, не обязательно плотной в $E$. Фиксируем число $T>0$ и выберем функцию $\varphi \in C([0, T])$, отличную от тождественного нуля.

Рассмотрим абстрактное дифференциальное уравнение:

$$
\frac{d u(t)}{d t}=A u(t)+\varphi(t) p, \quad 0 \leqslant t \leqslant T,
$$

с неизвестным элементом $p \in E$. Для одновременного нахождения $u(t), p$ зададим условия

$$
u(0)=u_{0}, \quad u(T)=u_{1}, \quad u_{0}, u_{1} \in D(A) .
$$

Указанную задачу (1), (2) будем назьвать обратной задачей.

Решением обратной задачи $(1),(2)$ считаем пару $(u(t), p)$, где $u:[0, T] \rightarrow E, p \in E$. $\Phi$ ункция $u(t)$ предполагается непрерьвно дифференцируемой в норме $E$ на отрезке $[0, T]$, ее значения принадлежат $D(A)$ при $0 \leqslant t \leqslant T$. Все соотношения $(1),(2)$ должны выполняться.

Обратная задача (1), (2) допускает разные интерпретации. Можно считать, например, что мы восстанавливаем в уравнении (1) неточно заданное неоднородное слагаемое $\varphi(t) p$ при помощи дополнительных "краевых" условий (2). Можно считать, что мы подбираем элемент $p \in E$ так, чтобы перевести систему из начального состояния $u_{0}$ в заданное финальное состояние $u_{1}$.

Работа первого автора вьполнена при поддержке Российского фонда фундаментальных исследований, грант № 00-01-00638. 
Подобные обратные задачи хорошо известны в литературе; обширная библиография имеется в [1]. Отметим, что в предыдущих работах исследования проводились при тех или иных ограничениях на оператор $A$. Например, предполагалось, что $A$ самосопряженньй оператор в гильбертовом пространстве [2]-[4]; что $A$ порождает соответствующую полугрупп [5]-[9]; что $A$ допускает манипуляции с преобразованием $\Phi$ рье [10], [11], и т.д.

Недавно авторы наметили новый подход. Выяснилось, что в вопросах единственности решения для обратной задачи $(1),(2)$ соображения, связанные с природой оператора $A$, могут быть не слишком существенны. В [12], [13] рассмотрен случай $\varphi(t) \equiv 1$; в [14]случай монотонного поведения $\varphi(t)$. Было показано, что в этих ситуациях единственность решения обратной задачи полностью определяется расположением на плоскости $\mathbb{C}$ собственных значений оператора $A$. Цель настоящей работы - распространить результаты [12]-[14] на общий случай функции $\varphi \in C([0, T])$.

Так как нас интересует единственность решения, заменим условия (2) однородньми условиями

$$
u(0)=0, \quad u(T)=0 .
$$

Обратная задача $(1),(3)$ всегда имеет тривиальное решение $u(t) \equiv 0, p=0$. Покажем, что при определенном выборе $A$ и $\varphi(t)$ обратная задача $(1),(3)$ будет заведомо иметь другие, нетривиальные решения.

Введем целую функцию

$$
\Phi(\lambda)=\int_{0}^{T} e^{-\lambda t} \varphi(t) d t, \quad \lambda \in \mathbb{C} .
$$

Функция $\Phi(\lambda)$ является конечным преобразованием Лапласа для $\varphi(t)$. Назовем $\Phi(\lambda)$ характеристической функиией обратной задачи $(1),(2)$ (и задачи $(1),(3))$. Так как $\varphi(t) \not \equiv 0$, то функция $\Phi(\lambda)(\not \equiv 0)$ имеет счетное множество различных нулей $\left\{\lambda_{k}\right\} \subset \mathbb{C}$ (см. $[15$, с. 107$])$. Обозначим это множество $\Lambda_{\Phi}$, т.е. $\Lambda_{\Phi}=\left\{\lambda_{k}\right\}$. Считаем, что нули занумерованы целочисленным индексом $k$ без учета кратности; система нумерации может зависеть от $\Phi(\lambda)$.

Предположим, что некоторый нуль $\lambda_{k} \in \Lambda_{\Phi}$ является собственным значением оператора $A$ с собственным вектором $f_{k}$, т.е. $A f_{k}=\lambda_{k} f_{k}$ для элемента $f_{k} \in D(A), f_{k} \neq 0$. Непосредственно проверяется, что пара

$$
u(t)=\int_{0}^{t} e^{\lambda_{k}(t-s)} \varphi(s) f_{k} d s, \quad p=f_{k}
$$

служит нетривиальњым решением однородной обратной задачи (1), (3). Следовательно, единственность решения неоднородной задачи $(1),(2)$ здесь нарушается.

Итак, для единственности решения обратной задачи необходимо потребовать, чтобы ни один нуль $\lambda_{k}$ характеристической функции (4) не являлся собственным значением оператора $A$. Выделим это предположение особо.

ОПРЕДЕЛЕНИЕ 1. Будем говорить, что в обратной задаче $(1),(2)$ (или в задаче (1), (3)) выполняется предположение $(\Lambda)$, если ни один нуль $\lambda_{k} \in \Lambda_{\Phi}$ характеристической функции $\Phi(\lambda)$ из формулы (4) не является собственным значением оператора $A$. 
Предположение $(\Lambda)$ необходимо для единственности решения задачи $(1),(2)$. Есть ситуации, когда оно оказьвается достаточным. Так будет в гильбертовом пространстве $E$ для самосопряженного полуограниченного оператора $A$ (см. [4]). Укажем на другой специальный случай, которьй понадобится нам в п. 3.

Теорема 1. Пусть $A: E \rightarrow E$ - линейный ограниченный оператор в банаховом пространстве E. Пусть $\varphi \in C([0, T])$ и $\varphi(t) \not \equiv 0$ на $[0, T]$. Если выполняется предположение $(\Lambda)$, то однородная обратная задача (1), (3) имеет только тривиальное решение $u(t) \equiv 0, p=0$.

ДокАЗАТЕЛЬСтво. Пусть выполнено предположение $(\Lambda)$, и $(u(t), p)$ - решение однородной обратной задачи $(1),(3)$. Запишем $u(t)$ через операторную экспоненту:

$$
u(t)=\int_{0}^{t} e^{A(t-s)} \varphi(s) p d s=e^{A t} \int_{0}^{t} e^{-A s} \varphi(s) p d s, \quad 0 \leqslant t \leqslant T
$$

Так как $u(T)=0$, то элемент $p$ принадлежит ядру оператора

$$
B=\int_{0}^{T} e^{-A s} \varphi(s) d s
$$

Применим операторное исчисление для линейных ограниченных операторов в банаховом пространстве $[16$, гл. $5, \S 1]$. Оператор $B$ совпадает с $\Phi(A)$ для целой функции $\Phi(\lambda)$ из формулы (4). Так как $\varphi(t) \not \equiv 0$ на $[0, T]$, то $\Phi(\lambda) \not \equiv 0$ в плоскости $\mathbb{C}$. По теореме об отображении спектра [16, теорема 5.12.2] оператор $B=\Phi(A)$ имеет ненулевое ядро тогда и только тогда, когда $\Phi(\alpha)=0$ для некоторого собственного значения $\alpha \in \mathbb{C}$ оператора $A$. Но в силу предположения $(\Lambda)$ ни один нуль $\lambda_{k}$ функции $\Phi(\lambda)$ не является собственным значением оператора $A$. Значит, оператор $B$ имеет нулевое ядро. Поэтому $p=0$ и $u(t) \equiv 0$ на $[0, T]$. Теорема доказана.

В общем случае линейного замкнутого оператора $A$ и произвольной непрерывной функции $\varphi(t) \not \equiv 0$ одно только предположение $(\Lambda)$ становится уже недостаточным для единственности решения обратной задачи $(1),(2)$.

ПримеР 1. Фиксируем $T>0$ и малое $\varepsilon>0$ так, чтобы $T-\varepsilon>0$. Рассмотрим модельную обратную задачу для уравнения в частных производных

$$
\left\{\begin{array}{l}
u_{t}(x, t)=-u_{x}(x, t)+\varphi(t) p(x), \quad 0 \leqslant x \leqslant \varepsilon, \quad 0 \leqslant t \leqslant T \\
u(0, t)=0, \\
u(x, 0)=0, \quad u(x, T)=0 .
\end{array}\right.
$$

Считаем, что $E=C([0, \varepsilon])$ и $A=-d / d x$ на области определения

$$
D(A)=\left\{f \in C^{1}([0, \varepsilon]): f(0)=0\right\} .
$$

Оператор $A$ не имеет собственньх значений в $E$. Предположение $(\Lambda)$ для него всегда выполняется. 
Выберем функцию $\varphi \in C([0, T])$ так, чтобы $\varphi(t)>0$ при $0 \leqslant t<T-\varepsilon$ и $\varphi(t) \equiv 0$ при $T-\varepsilon \leqslant t \leqslant T$. Пусть $p(x)$ - произвольная функция из $D(A)$, отличная от тождественного нуля. Определим для $(x, t) \in[0, \varepsilon] \times[0, T]$ функцию $u(x, t)$ по формуле

$$
u(x, t)= \begin{cases}\int_{0}^{t} \varphi(s) p(x-t+s) d s, & x-t \geqslant 0, \\ \int_{t-x}^{t} \varphi(s) p(x-t+s) d s, & x-t \leqslant 0 .\end{cases}
$$

Пара функций $(u(x, t), p(x))$ удовлетворяет соотношениям (6) и служит нетривиальным решением соответствующей абстрактной задачи (1), (3). При проверке равенства $u(x, T)=0$ надо учесть, что

$$
u(x, T)=\int_{T-x}^{T} \varphi(s) p(x-T+s) d s,
$$

где $\varphi(s) \equiv 0$ на $[T-x, T]$, ибо $[T-x, T] \subset[T-\varepsilon, T]$. Итак, для обратной задачи $(1),(3)$ в данной модели (6) имеет место неединственность решения, несмотря на выполнение предположения $(\Lambda)$.

Придадим идее примера другую, абстрактную формулировку.

Пусть $[0, T]$ - произвольный отрезок. Выберем функцию $\varphi(t) \not \equiv 0$ так, чтобы она обращалась в тождественньй нуль в малой окрестности $[T-\varepsilon, T]$ точки $t=T$. Выберем оператор $A$ так, чтобы он порождал $C_{0}$ полугруппу $U(t)$ со свойством $U(t) \equiv 0$ при $t \geqslant \varepsilon$. Такой оператор $A$ не имеет собственных значений в $E$ (см. [16, с. 552]), и предположение $(\Lambda)$ для него вьполняется. Решение неоднородной задачи Коши

записьвается через полугруппу:

$$
\frac{d u(t)}{d t}=A u(t)+\varphi(t) p, \quad 0 \leqslant t \leqslant T, \quad u(0)=0,
$$

$$
u(t)=\int_{0}^{t} U(t-s) \varphi(s) p d s=\int_{0}^{t} \varphi(s) U(t-s) p d s, \quad 0 \leqslant t \leqslant T .
$$

При $t=T$ имеем

$$
u(T)=\int_{0}^{T} \varphi(s) U(T-s) p d s .
$$

$\Phi$ ункция $U(T-s) p$ может быть отличной от нуля лишь при $T-\varepsilon \leqslant s \leqslant T$. Но здесь обращается в нуль функция $\varphi(s)$. Поэтому $u(T)=0$ при любом выборе элемента $p \neq 0$. Получаем бесконечно много нетривиальных решений однородной обратной задачи $(1),(3)$.

Нетрудно построить аналогичньй пример, основанный на обращении в нуль функции $\varphi(t)$ в окрестности точки $t=0$. Достаточно в предыдущем рассуждении сделать замену $t$ на $T-t$ и рассмотреть полученную задачу с оператором $A_{1}=-A$ и функцией $\varphi_{1}(t)=-\varphi(T-t)$.

Итак, если мы хотим для произвольного оператора $A$ охарактеризовать единственность решения обратной задачи $(1),(2)$ при помощи предположения $(\Lambda)$, то необходимо потребовать, чтобы функция $\varphi(t)$ не обращалась тождественно в нуль ни в одной из окрестностей точек $t=0$ и $t=T$. Используем краткую запись:

$$
\int_{0}^{\varepsilon}|\varphi(t)| d t>0, \quad \int_{T-\varepsilon}^{T}|\varphi(t)| d t>0 \quad \text { для любого малого } \varepsilon>0 .
$$

Будем называть условия (7) соотношениями невырожсденности на краях.

Теперь мы можем окончательно сформулировать интересующий нас 
Вопрос. Что нужно добавить к соотношениям (7) для того, чтобы единственность решения обратной задачи (1), (2) полностью выражалась предположением $(\Lambda)$ ?

Дальнейший план статьи следующий. В п. 2 выделены наиболее естественные условия, добавляемые к (7), при которых справедлив критерий единственности. Показано, что для функции $\varphi \in C([0, T])$ годится любое из предположений:

(i) $\varphi(0) \neq 0, \varphi(T) \neq 0$ (здесь (7) выполнено автоматически);

(ii) $\varphi(t)$ имеет ограниченную вариацию на $[0, T]$, и хотя бы одно из значений $\varphi(0)$ или $\varphi(T)$ отлично от нуля (на другом краю вьполняется соответствующее соотношение (7)).

В данньй момент мы не знаем, можно ли в условии (ii) отказаться от требования ограниченной вариации $\varphi(t)$ так, чтобы условие (i) стало не нужньм. Но возможно, что такой факт содержится в скрытой форме в следующем п. 3. Здесь получен общий критерий единственности решения, использующий понятие дефектного значения $d_{\varphi}$, определяемого функцией $\varphi(t)$. Для справедливости критерия достаточно предположения $d_{\varphi}<\infty$. Подсчет значения $d_{\varphi}$ нетривиален. Этот вопрос отдельно рассматривается в п. 4. Показано, что результаты п. 2 могут быть выведены из общего критерия, даже с определенными усилениями. Затем в п. 5 мы устанавливаем, что одних соотношений невырожденности (7) все же недостаточно для справедливости критерия единственности. Построен контрпример для функции $\varphi \in C^{\infty}([0, T])$, строго положительной в интервале $(0, T)$ и имеющей нули бесконечного порядка при $t=0$ и $t=T$. Здесь $d_{\varphi}=\infty$. Картина в определенном смысле замыкается. Пример использует идею предыдущей работы одного из авторов [14]. Некоторые особенности примера обсуждаются далее в п. 6. Последний п. 7 посвящен заключительньм замечаниям.

2. Критерий единственности - специальные случаи. Условия (i) и (ii), pacсматриваемые в данном пункте, выделены по следующим причинам. Во-первых, они удобны для приложений - их проверка элементарна. Во-вторых, прямое доказательство здесь более наглядно, и рассуждения данного пункта все равно потребуются в п. 4 для сведения теоремы 2 к общему случаю $d_{\varphi}<\infty$ (см. доказательство леммы 5$)$.

Сформулируем точньй результат.

ТЕОРемА 2. Пусть $A$ - линейный замкнутый оператор в банаховом пространстве Е. Пусть $\varphi \in C([0, T])$ и выполнены соотношения невырохсденности (7). Пусть, сверх того, выполняется любое из предположений:

(i) $\varphi(0) \neq 0, \varphi(T) \neq 0$;

(ii) функция $\varphi(t)$ имеет ограниченную вариачию на $[0, T]$, и хотя бы одно из значений $\varphi(0)$ или $\varphi(T)$ отлично от нуля.

Тогда для того чтобь однородная обратная задача (1), (3) имела только тривиальное решение $u(t) \equiv 0, p=0$, необходимо и достаточно, чтобы выполнялось предположсение $(\Lambda):$ ни один нуль $\lambda_{k} \in \Lambda_{\Phi}$ характеристической функиии $\Phi(\lambda)$ из формулы (4) не является собственным значением оператора $A$.

ДокАЗАТЕЛЬСТво. Утверждение о необходимости предположения $(\Lambda)$ установлено в п. 1. Действительно, если некоторый нуль $\lambda_{k} \in \Lambda_{\Phi}$ является собственньп значением оператора $A$ с собственным вектором $f_{k} \neq 0$, то формула (5) дает нетривиальное решение задачи (1), (3). 
Будем доказьвать достаточность. Пусть выполнено предположение $(\Lambda)$ : ни один нуль $\lambda_{k} \in \Lambda_{\Phi}$ характеристической функции $\Phi(\lambda)$ не является собственньм значением оператора $A$. Рассмотрим решение $(u(t), p)$ однородной обратной задачи $(1),(3)$ и покажем, что $u(t) \equiv 0, p=0$. Для удобства чтения и ссылок некоторые “замкнутые" пункты доказательства выделим в виде вспомогательных лемм.

ЛЕмма 1. Пусть $A$ - линейный замкнутый оператор в $E, u \varphi \in C([0, T])$. Пусть пара $(u(t), p)$ является решением однородной обратной задачи (1), (3). Введем характеристическую функиию $\Phi(\lambda)$ по формуле (4) и рассмотрим векторную челую функиию

$$
f(\lambda)=\int_{0}^{T} e^{-\lambda t} u(t) d t, \quad \lambda \in \mathbb{C} .
$$

Если для задачи (1), (3) выполнено предположсение $(\Lambda)$, то всякий нуль $\lambda_{k} \in \Lambda_{\Phi}$ характеристической функиии $\Phi(\lambda)$ является нулем не меньшей кратности для векторной челой функиии $f(\lambda)$.

ДокАЗАТЕЛЬСтво. Применим оператор $A$ к функции $f(\lambda)$ из формулы $(8)$ и воспользуемся тем, что $(u(t), p)$ - решение однородной обратной задачи $(1),(3)$. Имеем

$$
\begin{aligned}
A f(\lambda) & =\int_{0}^{T} e^{-\lambda t} A u(t) d t=\int_{0}^{T} e^{-\lambda t}\left(u^{\prime}(t)-\varphi(t) p\right) d t=\int_{0}^{T} e^{-\lambda t} u^{\prime}(t) d t-\Phi(\lambda) p \\
& =\lambda \int_{0}^{T} e^{-\lambda t} u(t) d t-\Phi(\lambda) p=\lambda f(\lambda)-\Phi(\lambda) p .
\end{aligned}
$$

Так как оператор $A$ замкнут, то полученное соотношение

$$
A f(\lambda)=\lambda f(\lambda)-\Phi(\lambda) p, \quad \lambda \in \mathbb{C}
$$

можно дифференцировать по $\lambda$. При последовательном дифференцировании имеем

$$
A f^{(k)}(\lambda)=\lambda f^{(k)}(\lambda)+k f^{(k-1)}(\lambda)-\Phi^{(k)}(\lambda) p, \quad \lambda \in \mathbb{C}, \quad k=1,2, \ldots
$$

Рассмотрим какой-нибудь нуль $\lambda_{k}$ характеристической функции $\Phi(\lambda)$. По предположению $(\Lambda)$ число $\lambda_{k}$ не является собственным значением оператора $A$. Подставим $\lambda=\lambda_{k}$ в соотношение (9). Видим, что $f\left(\lambda_{k}\right)=0$. Если $\lambda_{k}-$ кратньй нуль $\Phi(\lambda)$, то при подстановке в (10) заключаем по индукции, что $\lambda_{k}$ будет нулем не меньшей кратности для векторной целой функции $f(\lambda)$. Лемма 1 доказана.

Заменим теперь векторную ситуацию скалярной. Подействуем на функцию $f(\lambda)$ из формулы (8) линейньм непрерывным функционалом $f^{*} \in E^{*}$. Обозначим

$$
\Psi(\lambda)=f^{*}(f(\lambda)), \quad \psi(t)=f^{*}(u(t))
$$

Тогда $\Psi(\lambda)$ - обьгная целая функция переменной $\lambda \in \mathbb{C}$, а $\psi(t)$ - непрерьвная скалярная функция на $[0, T]$, причем

$$
\Psi(\lambda)=\int_{0}^{T} e^{-\lambda t} \psi(t) d t, \quad \lambda \in \mathbb{C}
$$


Более того, так как $u(t)$ - первая компонента решения $(u(t), p)$ однородной обратной задачи $(1),(3)$, то

$$
\psi \in C^{1}([0, T]), \quad \psi(0)=\psi(T)=0 .
$$

Для $\lambda \Psi(\lambda)$ получаем соотношение

$$
\lambda \Psi(\lambda) \equiv \lambda \int_{0}^{T} e^{-\lambda t} \psi(t) d t=\int_{0}^{T} e^{-\lambda t} \psi^{\prime}(t) d t, \quad \lambda \in \mathbb{C}
$$

с непрерьвной функцией $\psi^{\prime}(t)$.

Заметим, что при взятии функционала $f^{*}$ нули функции $f(\lambda)$ не исчезают и их кратности не уменьшаются. Поэтому при вьполненном предположении $(\Lambda)$ на основании леммы 1 всякий нуль $\lambda_{k} \in \Lambda_{\Phi}$ характеристической функции $\Phi(\lambda)$ будет нулем не меньшей кратности для функции $\Psi(\lambda)=f^{*}(f(\lambda))$. Иначе говоря, отношение

$$
Q(\lambda) \equiv \frac{\Psi(\lambda)}{\Phi(\lambda)}=\frac{\int_{0}^{T} e^{-\lambda t} \psi(t) d t}{\int_{0}^{T} e^{-\lambda t} \varphi(t) d t}
$$

определяет целую функцию переменной $\lambda \in \mathbb{C}$. Используя (13), получаем другую запись:

$$
Q(\lambda)=\frac{\lambda \Psi(\lambda)}{\lambda \Phi(\lambda)}=\frac{\int_{0}^{T} e^{-\lambda t} \psi^{\prime}(t) d t}{\lambda \int_{0}^{T} e^{-\lambda t} \varphi(t) d t} .
$$

Дальнейший анализ отношений $(14),(15)$ основан на известном "методе частных" [17, c. 540-542, с. 606-607]. Здесь мы во многом следуем работе [18]. Чтобы сделать изложение более ясным, приведем все основные моменты рассуждений. Наши формулировки не являются самыми общими - их лишь достаточно для доказательства основной теоремы 2. Нужные сведения из теории целых функций см. в [15], [17].

Лемма 2. Пусть $\varphi \in C([0, T])$ и выполнены соотношения невырохсденности (7). Пусть $\psi \in C([0, T])$ - другая функиия. Зададим преобразования Лапласа $\Phi(\lambda), \Psi(\lambda)$ по формулам (4), (11) соответственно. Предполохим, что их отношение (14) определяет иелую функиию $Q(\lambda)$. Тогда $Q(\lambda)$ есть функиия порядка не выше первого и минимального типа. Если, дополнительно, $|Q(\lambda)| \rightarrow 0$ при $|\lambda| \rightarrow \infty$ вдоль некоторой прямой в плоскости $\mathbb{C}$, то

$$
Q(\lambda) \equiv 0 \quad \text { nри всех } \quad \lambda \in \mathbb{C}
$$

В таком случае $\Psi(\lambda) \equiv 0$ при всех $\lambda \in \mathbb{C} u \psi(t) \equiv 0$ при всех $t \in[0, T]$.

ДокАЗАТЕЛЬСтвО. Рассмотрим индикаторы целых функций

$$
h_{\Phi}(\theta)=\varlimsup_{r \rightarrow \infty} \frac{\log \left|\Phi\left(r e^{i \theta}\right)\right|}{r}, \quad h_{\Psi}(\theta)=\varlimsup_{r \rightarrow \infty} \frac{\log \left|\Psi\left(r e^{i \theta}\right)\right|}{r}, \quad 0 \leqslant \theta<2 \pi .
$$

$\Phi$ ункции $\Phi(\lambda), \Psi(\lambda)$ являются конечньми преобразованиями Лапласа. Как отмечено в $[15$, с. $109-110]$, у их индикаторов для п.в. $\theta$ существует не только верхний, но 
и обычньй предел. Поэтому индикатор частного (14) равен разности индикаторов $h_{\Psi}(\theta)-h_{\Phi}(\theta)$. С помощью элементарных оценок проверяется, что

$$
h_{\Psi}(\theta) \leqslant \frac{T}{2}(|\cos \theta|-\cos \theta) \equiv \begin{cases}0, & \cos \theta \geqslant 0, \\ -T \cos \theta, & \cos \theta<0 .\end{cases}
$$

Для $\Phi(\lambda)$ справедливо равенство

$$
h_{\Phi}(\theta)=\frac{T}{2}(|\cos \theta|-\cos \theta)
$$

так как по условию функция $\varphi(t)$ удовлетворяет соотношениям невырожденности (7). Равенство (16) в предположении (7) доказано в классической работе [19, с. 287-290]; эквивалентное утверждение в терминах индикаторной диаграммы см. в [18, с. 96]; исчерпывающее изложение вопроса имеется в [15, с. 99-110].

Для индикатора целой функции $Q(\lambda)=\Psi(\lambda) / \Phi(\lambda)$ получаем

$$
h_{Q}(\theta)=h_{\Psi}(\theta)-h_{\Phi}(\theta) \leqslant \frac{T}{2}(|\cos \theta|-\cos \theta)-\frac{T}{2}(|\cos \theta|-\cos \theta)=0 .
$$

Максимум индикатора совпадает с типом целой функции $[17$, с. 98]. Поэтому функция $Q(\lambda)$ имеет порядок не выше первого и минимальньй тип. Всякая такая функция, ограниченная на некоторой прямой, есть тождественная константа [17, с. 71]. Константа, стремящаяся к нулю, есть тождественный нуль. Далее все очевидно. Лемма 2 доказана.

Покажем теперь, что в условиях теоремы 2 функция $Q(\lambda)$ ведет себя так, как надо для леммы 2 , в предположении (i) - на горизонтальной прямой $\operatorname{Im} \lambda=0$, а в предположении (ii) - на некоторой вертикальной прямой $\operatorname{Re} \lambda=a$.

Лемма 3. Пусть $\varphi \in C([0, T]), \varphi(0) \neq 0, \varphi(T) \neq 0$. Пусть $\psi \in C([0, T])$, $\psi(0)=\psi(T)=0$. Зададим преобразования Лапласа $\Phi(\lambda), \Psi(\lambda)$ по формулам (4), (11) соответственно и предполохсим, что их отношение (14) определяет иелую функиию $Q(\lambda)$. Тогда

$$
|Q(x)| \rightarrow 0 \quad \text { npu } \quad x \rightarrow \infty, \quad x \in \mathbb{R} .
$$

ДокАЗАТЕльство. Элементарно проверяется (см. также [20, с. 33]), что любая функция $\nu \in C([0, T])$ удовлетворяет соотношению

$$
x \int_{0}^{T} e^{-x t} \nu(t) d t \rightarrow \nu(0) \quad \text { при } \quad x \rightarrow+\infty .
$$

Отсюда при $x \rightarrow+\infty$ следует, что

$$
\begin{gathered}
Q(x)=\frac{x \Psi(x)}{x \Phi(x)}=\frac{x \int_{0}^{T} e^{-x t} \psi(t) d t}{x \int_{0}^{T} e^{-x t} \varphi(t) d t} \rightarrow \frac{\psi(0)}{\varphi(0)}=0 \\
Q(-x)=\frac{x e^{-x T} \Psi(-x)}{x e^{-x T} \Phi(-x)}=\frac{x \int_{0}^{T} e^{-x(T-t)} \psi(t) d t}{x \int_{0}^{T} e^{-x(T-t)} \varphi(t) d t}=\frac{x \int_{0}^{T} e^{-x s} \psi(T-s) d s}{x \int_{0}^{T} e^{-x s} \varphi(T-s) d s} \rightarrow \frac{\psi(T)}{\varphi(T)}=0 .
\end{gathered}
$$

Лемма 3 доказана. 
ЛЕмма 4. Пусть $\varphi \in C([0, T])$, причем $\varphi(t)$ имеет ограниченную вариацию на $[0, T]$ и хотя бы одно из значений $\varphi(0)$ или $\varphi(T)$ отлично от нуля. Пусть $\psi \in C^{1}([0, T]), \psi(0)=\psi(T)=0$. Зададим преобразования Лапласа $\Phi(\lambda), \Psi(\lambda)$ по формулам (4), (11) соответственно и предполоэим, что их отношение (14) определяет иелую функиию $Q(\lambda)$. Тогда существует такое $a \in \mathbb{R}$, что $|Q(a+i y)| \rightarrow 0$ при $y \rightarrow \infty, y \in \mathbb{R}$.

ДоКАЗАТЕЛЬСтвО. Пусть $\varphi(0) \neq 0$. Проинтегрируем по частям:

$$
\lambda \Phi(\lambda)=\lambda \int_{0}^{T} e^{-\lambda t} \varphi(t) d t=\varphi(0)-e^{-\lambda T} \varphi(T)+\int_{0}^{T} e^{-\lambda t} d \varphi(t) .
$$

Рассмотрим функцию $\lambda \Phi(\lambda)$ на некоторой прямой $\operatorname{Re} \lambda=a>0$. Разбив интеграл Стилтьеса на два, получим оценку

$$
\begin{aligned}
|\lambda \Phi(\lambda)| & \geqslant|\varphi(0)|-\left|e^{-\lambda T} \varphi(T)\right|-\left|\int_{0}^{\varepsilon} e^{-\lambda t} d \varphi(t)\right|-\left|\int_{\varepsilon}^{T} e^{-\lambda t} d \varphi(t)\right| \\
& \geqslant|\varphi(0)|-e^{-a T}|\varphi(T)|-\left.\operatorname{Var}\{\varphi(t)\}\right|_{0} ^{\varepsilon}-\left.e^{-a \varepsilon} \operatorname{Var}\{\varphi(t)\}\right|_{\varepsilon} ^{T} .
\end{aligned}
$$

Выберем $\varepsilon>0$ достаточно малым, а затем $a$ достаточно большим и добьемся того, чтобы на прямой $\operatorname{Re} \lambda=a$ вьполнялось соотношение $|\lambda \Phi(\lambda)| \geqslant \beta$ с некоторой константой $\beta>0$. Рассмотрим функцию $Q(\lambda)$ в записи (15). Пусть $\lambda=a+i y$. Тогда

$$
|Q(\lambda)|=\frac{|\lambda \Psi(\lambda)|}{|\lambda \Phi(\lambda)|} \leqslant \frac{1}{\beta}\left|\int_{0}^{T}\left(e^{-a t} \psi^{\prime}(t)\right) e^{-i y t} d t\right| \rightarrow 0 \quad \text { при } y \rightarrow \infty .
$$

Построенная прямая $\operatorname{Re} \lambda=a$ является искомой. Случай $\varphi(T) \neq 0$ легко сводится $\mathrm{k}$ предыдущему. Лемма 4 доказана.

Завершим доказательство теоремы 2. Напомним, что рассматривается функция $\psi(t)=f^{*}(u(t))$, где $(u(t), p)$ - решение однородной обратной задачи $(1),(3)$. Функция $\psi(t)$ удовлетворяет условиям (12). Исходя из предположения $(\Lambda)$, заключаем с учетом леммы 1 , что функция $Q(\lambda)$ в формуле $(14)$ является целой. Так как для $\varphi(t)$ выполняется условие (i) или (ii) теоремы 2 , то по соответствующей лемме 3 или 4 будем иметь, что $|Q(\lambda)| \rightarrow 0$ при $|\lambda| \rightarrow \infty$ вдоль некоторой прямой в плоскости $\mathbb{C}$. Отсюда на основании леммы 2 заключаем, что $\psi(t)=f^{*}(u(t)) \equiv 0$ на $[0, T]$. Выбор функционала $f^{*}$ был произвольным. По теореме Хана-Банаха $u(t) \equiv 0$ на $[0, T]$. Но тогда и $p=0$. Решение обратной задачи $(1),(3)$ оказьвается тривиальным. Теорема доказана.

Рассмотрим для примера следствие теоремы 2 для знакопостоянной функции $\varphi(t)$. Аналогичные утверждения отмечались ранее лишш в случае, когда к оператору $A$ была применима техника Фурье (см., например, [2], [10]). Другие достаточные признаки единственности, основанные на монотонности функции $\varphi(t)$, см. в [14].

СлЕДСТВИЕ. Пусть $A$ - линейный замкнутый оператор в Е без комплексных собственных значений (т.е. из равенства $A f=\lambda f$ следует либо $f=0$, либо $\lambda \in \mathbb{R})$. Пусть $\varphi \in C([0, T]), \varphi(t) \geqslant 0$ всюдуна $[0, T]$, причем $\varphi(0)>0, \varphi(T)>0$. Тогда однородная обратная задача (1), (3) имеет только тривиальное решение $u(t) \equiv 0, p=0$. 
ДокАЗАТЕЛЬСТвО. Функция $\varphi(t)$ удовлетворяет условиям теоремы 2 в части (i). Так как $\varphi(t) \geqslant 0$, то нули характеристической функции $\Phi(\lambda)$ являются комплексными (невещественными). Но у оператора $A$ нет комплексных собственных значений. По теореме 2 получаем требуемьй результат.

3. Критерий единственности: обобщение. Дадим обобщение критерия единственности в терминах некоторого дефектного значения $d_{\varphi}$, связанного с функцией $\varphi(t)$. Начнем с соответствующих определений.

Фиксируем отрезок $[0, T]$. Пусть $\varphi \in C([0, T]), \varphi(t) \not \equiv 0$ на $[0, T]$. Рассмотрим множество нулей $\Lambda_{\Phi}=\left\{\lambda_{k}\right\}$ преобразования Лапласа $\Phi(\lambda)$ из формулы (4). Пусть $m_{k}-$ кратность нуля $\lambda_{k}$. Сопоставим каждому нулю $\lambda_{k}$ показательную функцию $e^{-\lambda_{k} t}$. Если $m_{k} \geqslant 2$, добавим "присоединенные" функции $t e^{-\lambda_{k} t}, \ldots, t^{m_{k}-1} e^{-\lambda_{k} t}$. Построенную систему функций по всем нулям $\lambda_{k} \in \Lambda_{\Phi}$ обозначим $\mathscr{E}_{\varphi}$. Таким образом,

$$
\mathscr{E}_{\varphi}=\bigcup_{\lambda_{k} \in \Lambda_{\Phi}}\left\{e^{-\lambda_{k} t}, \ldots, t^{m_{k}-1} e^{-\lambda_{k} t}\right\}
$$

Система $\mathscr{E}_{\varphi}$ содержит все показательные и присоединенные к ним функции такие, что мера $\varphi(t) d t$ ортогональна системе $\mathscr{E} \varphi$.

Точнее понятие "ортогональности” вводится так. Для функции $\psi \in C([0, T])$ будем говорить, что мера $\psi(t) d t$ ортогональна системе $\mathscr{E}_{\varphi}$, если выполняются соотношения

$$
\int_{0}^{T} t^{j} e^{-\lambda_{k} t} \psi(t) d t=0, \quad \lambda_{k} \in \Lambda_{\Phi}, \quad j=0, \ldots, m_{k}-1
$$

Коротко записьваем так: $\psi(t) d t \perp \mathscr{E}_{\varphi}$.

Очевидно, что $\varphi(t) d t \perp \mathscr{E}_{\varphi}$. Рассмотрим линейное подпространство

$$
Z_{\varphi}=\left\{\psi \in C([0, T]): \psi(t) d t \perp \mathscr{E}_{\varphi}\right\}, \quad Z_{\varphi} \subset C([0, T])
$$

Пространство $Z_{\varphi}$ является неким “ортогональным дополнением” к системе E् (порожденной, в свою очередь, функцией $\varphi(t))$. Для характеристики размерности ортогонального дополнения используют понятие “дефекта". Это объясняет следующую терминологию.

ОПРЕДЕЛЕНИЕ 2. Дефектным значением функции $\varphi(t)$ назовем величину

$$
d_{\varphi} \equiv \operatorname{dim} Z_{\varphi}
$$

(линейную размерность пространства $Z_{\varphi}$ ).

Вопрос о вычислении $d_{\varphi}$ обсуждается далее в п. 4. Сейчас лишь отметим, что $1 \leqslant$ $d_{\varphi} \leqslant \infty$. Соотношение $d_{\varphi} \geqslant 1$ очевидно, ибо $\varphi \in Z_{\varphi}$ и, значит, $Z_{\varphi} \neq\{0\}$. Соотношение $d_{\varphi}=\infty$ не исключается (см. п. 4). Особо подчеркнем, что понятие дефектного значения $d_{\varphi}$ привязано к фиксированному отрезку $[0, T]$, изменение которого не допускается.

Самого определения $d_{\varphi}$ достаточно для доказательства следующего критерия. 
ТеОрема 3. Пусть $A$ - линейный замкнутый оператор в банаховом пространстве Е. Пусть $\varphi \in C([0, T]), \varphi(t) \not \equiv 0$ на $[0, T]$. Предположим, что

$$
d_{\varphi} \equiv \operatorname{dim} Z_{\varphi}<\infty .
$$

Тогда для того чтобы однородная обратная задача (1), (3) имела только тривиальное решение $u(t) \equiv 0, p=0$, необходимо и достаточно, чтобы выполнялось предполохсение $(\Lambda):$ ни один нуль $\lambda_{k} \in \Lambda_{\Phi}$ характеристической функиии $\Phi(\lambda)$ из формуль (4) не является собственным значением оператора $A$.

ДокАЗАТЕльство. Необходимость получается так же, как в теореме 2 , ссылкой на формулу (5). Доказьваем достаточность. Пусть ни один нуль $\lambda_{k} \in \Lambda_{\Phi}$ характеристической функции $\Phi(\lambda)$ не является собственным значением оператора $A$. Рассмотрим решение $(u(t), p)$ однородной обратной задачи $(1),(3)$ и покажем, что $u(t) \equiv 0, p=0$.

Для функционала $f^{*} \in E^{*}$ положим $\psi(t) \equiv f^{*}(u(t))$. Тогда $\psi \in C([0, T])$. По формуле $(11)$ введем преобразование Лапласа $\Psi(\lambda)$. Так же, как в п. 2 , из леммы 1 следует, что всякий нуль $\lambda_{k}$ характеристической функции $\Phi(\lambda)$ будет нулем не меньшей кратности для $\Psi(\lambda)$. Поэтому $\psi(t) d t \perp \mathscr{E}_{\varphi}$. Но тогда функция $\psi(t)=f^{*}(u(t))$ принадлежит $Z_{\varphi}$ при любом выборе функционала $f^{*} \in E^{*}$. По условию $d_{\varphi} \equiv \operatorname{dim} Z_{\varphi}<\infty$. Вьведем отсюда, что функция $u(t)$ является конечномерной, т. е. что все ее значения принадлежат конечномерному подпространству пространства $E$.

Фиксируем подпространство $E_{0} \equiv \operatorname{span}\{u(t): 0 \leqslant t \leqslant T\}$ - линейную оболочку значений функции $u(t)$ (множество конечных линейных комбинаций, составленных из этих значений). Тогда $u(t) \in E_{0} \subset E$ при каждом $t \in[0, T]$. Из определения $E_{0}$ вытекает также включение $E_{0} \subset D(A)$. Покажем, что $\operatorname{dim} E_{0} \leqslant d_{\varphi}$.

Пусть $\operatorname{dim} E_{0}>d_{\varphi} \equiv d$. Тогда существуют элементы $u\left(t_{1}\right), \ldots, u\left(t_{d}\right), u\left(t_{d+1}\right)$, линейно независимые в пространстве $E$. Рассмотрим биортогональную систему функционалов $f_{1}^{*}, \ldots, f_{d}^{*}, f_{d+1}^{*} \in E^{*}$ :

$$
f_{k}^{*}\left(u\left(t_{j}\right)\right)=\delta_{k j}, \quad k, j=1, \ldots, d, d+1 .
$$

Функции $\psi_{k}(t)=f_{k}^{*}(u(t)), k=1, \ldots, d, d+1$, принадлежат $Z_{\varphi}$ и являются линейно зависимыми, ибо $\operatorname{dim} Z_{\varphi}=d_{\varphi} \equiv d$. Следовательно, существует набор констант $\alpha_{1}, \ldots, \alpha_{d}, \alpha_{d+1}$ такой, что $\sum\left|\alpha_{k}\right|>0$ и

$$
\alpha_{1} f_{1}^{*}(u(t))+\cdots+\alpha_{d} f_{d}^{*}(u(t))+\alpha_{d+1} f_{d+1}^{*}(u(t)) \equiv 0, \quad 0 \leqslant t \leqslant T .
$$

Подставляя значения $t=t_{j}$, получаем $\alpha_{j}=0$ для $j=1, \ldots, d, d+1$. Противоречие с условием $\sum\left|\alpha_{k}\right|>0$ показьвает невозможность допущения $\operatorname{dim} E_{0}>d_{\varphi}$.

Итак, $\operatorname{dim} E_{0} \leqslant d_{\varphi}<\infty$. Значения функции $u(t)$ принадлежат конечномерному подпространству $E_{0}$ пространства $E$. В силу конечномерности подпространство $E_{0}$ замкнуто в $E$ и, значит, этому же подпространству принадлежат значения производной $u^{\prime}(t)$. Рассмотрим теперь элемент $p$ - второй компонент решения $(u(t), p)$ обратной задачи (1), (3). Покажем, что $p \in E_{0}$.

Вновь будем рассуждать “от противного". Пусть $p \notin E_{0}$. Тогда $p \neq 0$ и, значит, $u(t) \not \equiv 0$ на $[0, T]$. Поэтому $\operatorname{dim} E_{0}=n$ для $1 \leqslant n \leqslant d_{\varphi}$. Выберем базис $e_{1}, \ldots, e_{n}$ в $E_{0}$. Система векторов $e_{1}, \ldots, e_{n}, p$ линейно независима в $E$. Рассмотрим систему функционалов $e_{1}^{*}, \ldots, e_{n}^{*}, p^{*} \in E^{*}$, биортогональную к системе $e_{1}, \ldots, e_{n}, p$. Положим 
$\nu_{k}(t)=e_{k}^{*}(u(t)), k=1, \ldots, n$. Все функции $\nu_{k}(t)$ непрерывно дифференцируемы на $[0, T], \nu_{k}^{\prime}(t)=e_{k}^{*}\left(u^{\prime}(t)\right)$. Запишем разложения по базису:

$$
u(t)=\nu_{1}(t) e_{1}+\cdots+\nu_{n}(t) e_{n}, \quad u^{\prime}(t)=\nu_{1}^{\prime}(t) e_{1}+\cdots+\nu_{n}^{\prime}(t) e_{n} .
$$

Подставим разложения (18) в соотношения (1), (3). Имеем

$$
\left\{\begin{array}{l}
\sum_{k=1}^{n} \nu_{k}^{\prime}(t) e_{k}=\sum_{k=1}^{n} \nu_{k}(t) A e_{k}+\varphi(t) p, \quad 0 \leqslant t \leqslant T \\
\nu_{1}(0) e_{1}+\cdots+\nu_{n}(0) e_{n}=\nu_{1}(T) e_{1}+\cdots+\nu_{n}(T) e_{n}=0 .
\end{array}\right.
$$

Подействуем на полученную систему функционалами $e_{1}^{*}, \ldots, e_{n}^{*}$. Для краткости обозначим $a_{j k}=e_{j}^{*}\left(A e_{k}\right)$. Имеем набор соотношений

$$
\left\{\begin{array}{l}
\nu_{j}^{\prime}(t)=\sum_{k=1}^{n} a_{j k} \nu_{k}(t)+0, \quad 0 \leqslant t \leqslant T, \quad j=1, \ldots, n, \\
\nu_{j}(0)=\nu_{j}(T)=0, \quad j=1, \ldots, n .
\end{array}\right.
$$

Соотношения (19) представляют собой линейную однородную систему ОДУ с нулевыми граничными условиями на обоих краях. Ясно, что $\nu_{j}(t) \equiv 0$ на $[0, T]$ для $j=1, \ldots, n$. Но тогда из (18) следует, что $u(t) \equiv 0$ на $[0, T]$. Подставив в уравнение $(1)$, получаем $p=0$, что противоречит допущению $p \notin E_{0}$. Значит, допущение неверно, и $p \in E_{0}$.

Итак, $u(t), u^{\prime}(t), p \in E_{0}$. Но тогда $A u(t)=u^{\prime}(t)-\varphi(t) p \in E_{0}$ при $0 \leqslant t \leqslant T$. Отсюда $A\left(E_{0}\right) \subset E_{0}$, т.е. подпространство $E_{0}=\operatorname{span}\{u(t)\}$ инвариантно относительно оператора $A$. Обозначим $\left.A_{0} \equiv A\right|_{E_{0}}$ - сужение оператора $A$ на конечномерное подпространство $E_{0}$. Оператор $A_{0}: E_{0} \rightarrow E_{0}$ ограничен, предположение $(\Lambda)$ для него сохраняется. Пара $(u(t), p)$ будет решением конечномерной обратной задачи

$$
\frac{d u(t)}{d t}=A_{0} u(t)+\varphi(t) p, \quad 0 \leqslant t \leqslant T, \quad u(0)=u(T)=0,
$$

рассматриваемой в пространстве $E_{0}$. По теореме 1 обратная задача $(20)$ с ограниченным оператором $A_{0}$ имеет только тривиальное решение $u(t) \equiv 0, p=0$. Теорема доказана.

Для применения полученной теоремы 3 надо рассмотреть вопрос о вычислении дефектного значения $d_{\varphi}$.

4. О дефектном значении $d_{\varphi}$. Всюду по-прежнему считаем, что $\varphi \in C([0, T])$, $\varphi(t) \not \equiv 0$ на $[0, T]$. Понятия $\Phi(\lambda), \mathscr{E}_{\varphi}, Z_{\varphi}, d_{\varphi}$ определяем, как в п. 3. Для произвольной функции $\psi \in C([0, T])$ вводим преобразование Лапласа $\Psi(\lambda)$ по формуле (11). Из определения $Z_{\varphi}$ легко выводится, что $\psi \in Z_{\varphi}$ тогда и только тогда, когда всякий нуль $\lambda_{k} \in \Lambda_{\Phi}$ функции $\Phi(\lambda)$ является нулем не меньшей кратности для функции $\Psi(\lambda)$. Другими словами, отношение $\Psi(\lambda) / \Phi(\lambda)$ должно определять целую функцию $Q(\lambda)$. Этот удобный критерий принадлежности $\psi(t)$ пространству $Z_{\varphi}$ используется далее без оговорок.

Сопоставим полученные теоремы 2 и 3 с точки зрения дефектного значения $d_{\varphi}$. 
ЛЕмма 5. Пусть $\varphi \in C([0, T])$ и выполнены соотношения невырохсденности (7). Пусть, сверх того, выполняется любое из предположений (i) или (ii) теоремы 2. Тогда $d_{\varphi} \equiv \operatorname{dim} Z_{\varphi}=1$.

ДокАЗАТЕЛЬСтво. Предположим, что $d_{\varphi}>1$. Значит, пространство $Z_{\varphi}$ содержит функцию $\psi \in C([0, T])$, линейно независимую с функцией $\varphi(t)$. Отношение преобразований Лапласа $\Psi(\lambda) / \Phi(\lambda)$ будет определять целую функцию $Q(\lambda)$, отличную от тождественной константы. По лемме 2 функция $Q(\lambda)$ имеет порядок не выше первого и минимальный тип. У такой функции заведомо есть нули (например, на основании классической теоремы Адамара $[17$, с. 38$])$. Пусть $\lambda_{0}-$ нуль функции $Q(\lambda)$. Тогда $\lambda_{0}$ будет нулем функции $\Psi(\lambda)=Q(\lambda) \Phi(\lambda)$. Определим новую функцию

$$
\psi_{1}(t)=\int_{0}^{t} e^{\lambda_{0}(t-s)} \psi(s) d s, \quad 0 \leqslant t \leqslant T
$$

Здесь $\psi_{1} \in C^{1}([0, T]), \psi_{1}(0)=\psi_{1}(T)=0$, т.е. для функции $\psi_{1}(t)$ вьполнены соотношения (12). (Равенство $\psi_{1}(T)=0$ следует из того, что $\lambda_{0}$ есть нуль функции $\Psi(\lambda)$.) Рассмотрим преобразование Лапласа:

$$
\Psi_{1}(\lambda)=\int_{0}^{T} e^{-\lambda t} \psi_{1}(t) d t=\int_{0}^{T} e^{-\lambda t}\left(\int_{0}^{t} e^{\lambda_{0}(t-s)} \psi(s) d s\right) d t
$$

После элементарных подсчетов получим $\Psi_{1}(\lambda)=\Psi(\lambda) /\left(\lambda-\lambda_{0}\right)$. Таким образом, отношение $Q_{1}(\lambda) \equiv \Psi_{1}(\lambda) / \Phi(\lambda)=Q(\lambda) /\left(\lambda-\lambda_{0}\right)$ есть целая функция переменной $\lambda$. Теперь в предположении (i) воспользуемся леммой 3 , а в предположении (ii) - леммой 4. Применим эти леммы к функции $\psi_{1}(t)$, после чего по лемме $2 \psi_{1}(t) \equiv 0$ на $[0, T]$. Но тогда $\psi(t)=\psi_{1}^{\prime}(t)-\lambda_{0} \psi_{1}(t) \equiv 0$ на $[0, T]$, что противоречит выбору $\psi(t)$. Следовательно, предположение $d_{\varphi}>1$ невозможно, и $d_{\varphi}=1$. Лемма доказана.

Из леммы 5 следует, что теорема 2 включается в общую теорему 3 на правах частного случая с $d_{\varphi}=1$. Покажем, что с помощью теоремы 3 можно еще ослабить условие (i) теоремы 2. Воспользуемся одним результатом Любича [18, с. 101], изучавшего вопрос о вьгислении дефектного значения $d_{\varphi}$ в болееширокой (необязательной для наших целей) постановке. Получим следующее утверждение для обратной задачи.

ТЕОРемА 4. Пусть $A$ - линейный замкнутый оператор в банаховом пространстве Е. Пусть $\varphi \in C([0, T])$ и выполнены соотношения

$$
\varphi(t) \sim C_{0} t^{\alpha_{0}}, \quad \varphi(T-t) \sim C_{1} t^{\alpha_{1}} \quad n p u \quad t \rightarrow 0+
$$

с константами $C_{k} \neq 0, \alpha_{k} \geqslant 0$ для $k=0,1$. Тогда $d_{\varphi}<\infty$ и однородная обратная задача (1), (3) удовлетворяет требованиям теоремы 3. Следовательно, для того чтобь обратная задача (1), (3) имела только тривиальное решение, необходимо и достаточно, чтобы выполнялось предположение $(\Lambda)$. 
ДокАЗАТЕЛЬСтво. Надо показать, что $d_{\varphi}<\infty$. Сведем ситуацию к схеме работы [18]. Рассмотрим функцию

$$
\mu(t)=\int_{0}^{t} \varphi(s) d s, \quad 0 \leqslant t \leqslant T .
$$

По правилу Лопиталя из (21) вьводятся соотношения

$$
\mu(t)-\mu(0) \sim \frac{C_{0}}{\alpha_{0}+1} t^{\alpha_{0}+1}, \quad \mu(T)-\mu(T-t) \sim \frac{C_{1}}{\alpha_{1}+1} t^{\alpha_{1}+1}, \quad t \rightarrow 0+.
$$

Применим к мере $d \mu(t)=\varphi(t) d t$ теорему 3 работы [18]. Получим, что ортогональное дополнение $\mathscr{E}_{\varphi}^{\perp}$ к системе $\mathscr{E}_{\varphi}$ в пространстве $(C([0, T]))^{*}$ имеет конечную размерность, не превосходящую меньшего из чисел $\left[\alpha_{0}\right]+2$ или $\left[\alpha_{1}\right]+2$. Пространство $Z_{\varphi}$ вкладывается в $\mathscr{E}_{\varphi}^{\perp}$ при отображении $\psi \rightarrow \psi(t) d t$. Следовательно, $d_{\varphi} \equiv \operatorname{dim} Z_{\varphi} \leqslant \operatorname{dim} \mathscr{E} \perp<\infty$. Остальное очевидно. Теорема доказана.

Итак, если на краях - при $t=0$ и $t=T$ - функция $\varphi(t)$ имеет нули не вьше конечного порядка, то $d_{\varphi}<\infty$. Такие функции образуют весьма широкий класс, удобный для приложений. Но случай $d_{\varphi}=\infty$ тоже не исключается. Так будет, например, всякий раз, когда для $\varphi(t)$ не выполняется хотя бы одно соотношение невырожденности (7). Это нетрудно заметить, сравнивая вьводы примера 1 и теорему 3. Для полноты изложения приведем явньй пример, не ссылающийся на предыдушие результаты.

ПРимеР 2. Пусть функция $\varphi(t)$ не удовлетворяет какому-то условию (7), например, при $t=T$. Тогда $\varphi(t) \equiv 0$ на промежутке $[T-\varepsilon, T]$ с некоторым малым $\varepsilon>0$. Для $h \in(0, \varepsilon)$ рассмотрим функцию

$$
\psi_{h}(t)= \begin{cases}\int_{0}^{t} \varphi(s) d s, & 0 \leqslant t \leqslant h \\ \int_{t-h}^{t} \varphi(s) d s, & h \leqslant t \leqslant T .\end{cases}
$$

Непрерьвность $\psi_{h}(t)$ на $[0, T]$ очевидна. Вычислим преобразование Лапласа:

$$
\Psi_{h}(\lambda)=\int_{0}^{T} e^{-\lambda t} \psi_{h}(t) d t=\int_{0}^{h} e^{-\lambda t}\left(\int_{0}^{t} \varphi(s) d s\right) d t+\int_{h}^{T} e^{-\lambda t}\left(\int_{t-h}^{t} \varphi(s) d s\right) d t .
$$

После элементарных подсчетов с учетом того, что $\varphi(t) \equiv 0$ на $[T-h, T]$, получим

$$
\Psi_{h}(\lambda)=\frac{1-e^{-h \lambda}}{\lambda} \Phi(\lambda) .
$$

Отношение $\Psi_{h}(\lambda) / \Phi(\lambda)$ определяет целую функцию $\left(1-e^{-h \lambda}\right) / \lambda$. Поэтому $\psi_{h} \in Z_{\varphi}$ при любом $h \in(0, \varepsilon)$. Функций $\psi_{h}(t)$ бесконечно много. Все они линейно независимы в $C([0, T])$. Последнее следует, например, из того, что правая граница носителя функции $\psi_{h}(t)$ сдвигается вправо при росте $h$. Таким образом, пространство $Z_{\varphi}$ бесконечномерно и $d_{\varphi} \equiv \operatorname{dim} Z_{\varphi}=\infty$. Аналогично рассматривается случай, когда $(7)$ не вьполняется при $t=0$.

Итак, соотношения невырожденности (7) необходимы для $d_{\varphi}<\infty$. Необходимы, но еще не достаточны. 
ПримеР 3 (Любич $\left[18\right.$, с. 95]). Пусть $\varphi \in C^{\infty}([0, T]), \varphi(t)>0$ при $0<t<T$ и $\varphi^{(n)}(0)=\varphi^{(n)}(T)=0$ при $n=0,1,2, \ldots$ Соотношения (7) здесь вьполняются. Отношение преобразования Лапласа для $\varphi^{(n)}(t)$ к преобразованию Лапласа для $\varphi(t)$ есть целая функция $\lambda^{n}$, что следует из очевидного тождества

$$
\int_{0}^{T} e^{-\lambda t} \varphi^{(n)}(t) d t=\lambda^{n} \int_{0}^{T} e^{-\lambda t} \varphi(t) d t .
$$

Поэтому пространство $Z_{\varphi}$ кроме самой функции $\varphi(t)$ содержит все ее производные. $\Phi$ ункции $\varphi(t), \varphi^{\prime}(t), \ldots, \varphi^{(n)}(t), \ldots$ линейно независимы в пространстве $C([0, T])$, так как в противном случае функция $\varphi(t)$ была бы решением линейного однородного ОДУ с нулевыми условиями Коши. Таким образом, $d_{\varphi} \equiv \operatorname{dim} Z_{\varphi}=\infty$.

Ключевая особенность примера 3 состоит в том, что на краях - при $t=0$ и $t=T$ - функция $\varphi(t)$ имеет нули бесконечного порядка. Такие функции не подпадают под действие теоремы 4. Это неслучайное обстоятельство. Покажем, что для обратной задачи $(1),(2)$ с функцией $\varphi(t)$ из примера 3 единственность решения может уже не выражаться предположением $(\Lambda)$.

5. Пример обратной задачи для $d_{\varphi}=\infty$. Рассмотрим банахово пространство $E=C([0,1])$ с обычной sup-нормой. Фиксируем натуральное $N \geqslant 2$ и зададим оператор $A=d^{N} / d x^{N}$ на области определения

$$
D(A)=\left\{f \in C^{N}([0,1]): f(0)=f^{\prime}(0)=\cdots=f^{(N-1)}(0)=0\right\} .
$$

Непосредственно проверяется, что $A$ - линейный замкнутьй оператор в $E$, не имеющий собственных значений. Его резольвента $(\lambda-A)^{-1}$ определена всюду в $\mathbb{C}$. Запишем через свертку:

$$
(\lambda-A)^{-1} g(x)=-\int_{0}^{x} y(x-s, \lambda) g(s) d s=-\int_{0}^{x} y(s, \lambda) g(x-s) d s, \quad g \in E,
$$

где $y(x, \lambda)$ - решение задачи Коши

$$
\frac{d^{N} y}{d x^{N}}=\lambda y, \quad y(0)=\cdots=y^{(N-2)}(0)=0, \quad y^{(N-1)}(0)=1,
$$

с параметром $\lambda \in \mathbb{C}$. Функция $y(x, \lambda)$ выражается в виде

$$
y(x, \lambda)=\frac{x^{N-1}}{(N-1) !}+\lambda \frac{x^{2 N-1}}{(2 N-1) !}+\lambda^{2} \frac{x^{3 N-1}}{(3 N-1) !}+\cdots=x^{N-1} Y_{N}\left(\lambda x^{N}\right),
$$

где

$$
Y_{N}(z)=\frac{1}{(N-1) !}+\frac{z}{(2 N-1) !}+\frac{z^{2}}{(3 N-1) !}+\cdots=\sum_{m=0}^{\infty} \frac{z^{m}}{\Gamma(m N+N)}, \quad z \in \mathbb{C} .
$$

$\Phi$ ункция $Y_{N}(z)$ совпадает с целой функцией типа Миттаг-Леффлера $E_{1 / N}(z ; N)$, имеющей порядок $\rho=1 / N<1$ (см. [21]).

Выберем функцию $\varphi(t)$ на некотором отрезке $[0, T]$ со следующими свойствами:

1) $\varphi \in C^{\infty}([0, T]), \varphi(t)>0$ на $(0, T), \varphi^{(k)}(0)=\varphi^{(k)}(T)=0$ при $k=0,1,2, \ldots$;

$2)$ производные функции $\varphi(t)$ удовлетворяют оценкам

$$
\left|\varphi^{(k)}(t)\right| \leqslant 2^{k}(k !)^{3 / 2}, \quad k=0,1,2, \ldots, \quad 0 \leqslant t \leqslant T .
$$


Существование таких $\varphi(t)$ доказьвается в теории квазианалитических функций. Можно, например, воспользоваться результатом Хёрмандера [22, с. 32]. (Чтобы получить наш случай, надо в теореме 1.3 .5 [22] положить $a_{0}=1, a_{k}=k^{-3 / 2}$ для всех натуральных $k$.)

Оператор $A$ и функция $\varphi(t)$ соответствуют поставленньм целям. Оператор $A$ не имеет собственных значений, и предположение $(\Lambda)$ заведомо выполняется. Функция $\varphi(t)$ подчинена соотношениям невырожденности $(7)$, но $d_{\varphi}=\infty$ (см. пример 3 в п. 4). Запишем обратную задачу (1), (3) для указанных $A, \varphi(t)$ в виде

$$
\left\{\begin{array}{l}
u_{t}(x, t)=u_{x^{N}}(x, t)+\varphi(t) p(x), \quad 0 \leqslant x \leqslant 1, \quad 0 \leqslant t \leqslant T, \\
u(0, t)=u_{x}(0, t)=\cdots=u_{x^{N-1}}(0, t)=0, \\
u(x, 0)=0, \quad u(x, T)=0 .
\end{array}\right.
$$

Рассмотрим пару функций

$$
u(x, t)=\frac{x^{N}}{N !} \varphi(t)+\frac{x^{2 N}}{(2 N) !} \varphi^{\prime}(t)+\cdots+\frac{x^{(k+1) N}}{((k+1) N) !} \varphi^{(k)}(t)+\cdots, \quad p(x) \equiv-1 .
$$

Сходимость ряда для $u(x, t)$ и возможность почленного дифференцирования следуют из оценок (24). Подстановка пары (26) в (25) дает верные тождества. Таким образом, несмотря на отсутствие собственных значений у оператора $A$, обратная задача (25) имеет нетривиальное решение $(u(x, t), p(x))$.

6. Дополнения к примеру. При $N=2$ пример п. 5 указан в [14] без обсуждения дефектного значения $d_{\varphi}$. Ряд, выражающий $u(x, t)$ в $(26)$, напоминает известную конструкцию А.Н. Тихонова, использованную в [23] с иной целью: показать неединственность решения задачи Коши для уравнения теплопроводности.

Отметим, что при $N \geqslant 2$ оператор $A=d^{N} / d x^{N}$ с областью определения (22) обладает интересной особенностью: однородное уравнение $d u(t) / d t=A u(t)$ на любом промежутке $[0, T]$ имеет только тривиальное решение $u(t) \equiv 0$. При $N=2$ данньй факт также упомянут в $\left[23\right.$, с. 201], где рассматривалось уравнение $u_{t}=u_{x \boldsymbol{x}}$ при условиях $u(0, t)=u_{x}(0, t)=0$. Кроме того, понятна связь с теоремой Хольмгрена [24, с. 259]. Эти классические результаты основаны на технике "уравнений с частньми производными". Другое, “операторное” доказательство равенства $u(t) \equiv 0$ можно получить, применяя в нашей ситуации результаты Любича и Ткаченко [25, c. 430-433] о решениях уравнения $d u(t) / d t=A u(t)$ с оператором $A$, имеющим мероморфную резольвенту. Но методы работы [25] довольно сложны и требуют специальной теории “локального преобразования Лапласа".

Дадим сейчас новое, очень короткое доказательство тривиальности решения $u(t)$ в уравнении $d u(t) / d t=A u(t)$ с оператором $A=d^{N} / d x^{N}, N \geqslant 2$, на области определения $(22)$ в пространстве $E=C([0,1])$. Пространство $C([0,1])$ взято, конечно, лишь для удобства; аналогично можно рассмотреть $C([0, l]), L^{p}([0, l])$ и т.д. Решение уравнения $d u(t) / d t=A u(t)$ понимается в классическом смысле, как функция $u:[0, T] \rightarrow E$ из класса $C^{1}([0, T] ; E)$ со значениями в $D(A)$.

Пусть $u(t)$ - такое решение. При всех $\lambda \in \mathbb{C}$ справедливо равенство

$$
A \int_{0}^{T} e^{-\lambda t} u(t) d t=\int_{0}^{T} e^{-\lambda t} u^{\prime}(t) d t
$$


Проинтегрируем правую часть по частям и запишем в виде

$$
(\lambda-A) \int_{0}^{T} e^{-\lambda t} u(t) d t=u(0)-e^{-\lambda T} u(T)
$$

Отсюда

$$
\int_{0}^{T} e^{-\lambda t} u(t) d t=(\lambda-A)^{-1}\left(u(0)-e^{-\lambda T} u(T)\right), \quad \lambda \in \mathbb{C} .
$$

Резольвента $(\lambda-A)^{-1}$ определена при $\lambda \in \mathbb{C}$ формулой $(23)$ с функцией $y(x, \lambda)=$ $x^{N-1} Y_{N}\left(\lambda x^{N}\right)$. Напомним, что $Y_{N}(z)=E_{1 / N}(z ; N)$ - целая функция порядка $1 / N$. $\mathrm{C}$ помощью элементарных оценок получаем, что сама резольвента $(\lambda-A)^{-1}$ является операторной целой функцией от $\lambda$ порядка $\rho \leqslant 1 / N<1$.

Подставим в (27) значения $\lambda=\zeta_{k} \equiv 2 k \pi i / T, k \in \mathbb{Z}$. Имеем

$$
\left(\zeta_{k}-A\right)^{-1}(u(0)-u(T))=\int_{0}^{T} e^{-(2 k \pi i / T) t} u(t) d t \rightarrow 0 \quad \text { при } \quad k \rightarrow \infty
$$

Рассмотрим векторную целую функцию $v(\lambda) \equiv(\lambda-A)^{-1}(u(0)-u(T))$. Порядок $v(\lambda)$ не превосходит $1 / N$ и согласно $(28)\left|v\left(\zeta_{k}\right)\right| \rightarrow 0$ при $k \rightarrow \infty$. Точки $\zeta_{k}=2 k \pi i / T$ образуют двустороннюю арифметическую прогрессию. На основании известной теоремы Полиа $\left[16\right.$, с. 118] заключаем, что $v(\lambda) \equiv 0$ в плоскости $\mathbb{C}$. В частности, $v\left(\zeta_{k}\right)=0$ для всех $k \in \mathbb{Z}$. Вновь обращаясь к $(28)$, видим, что функция $u(t)$ ортогональна на $[0, T]$ всем экспонентам $e^{(2 k \pi i / T) t}, k \in \mathbb{Z}$. Поэтому $u(t) \equiv 0$ на $[0, T]$. Требуемое получено.

Отсутствие нетривиальных решений у однородного уравнения $d u(t) / d t=A u(t)$ не означает, конечно, “тривиальности” самого оператора $A$. Как показьвает пример в п. 5 , неоднородное уравнение $d u(t) / d t=A u(t)+\varphi(t) p$ при соответствующем выборе $\varphi(t)$ имеет нетривиальное "решение" (26), представляюшее интерес для обратных задач.

7. Заключительные замечания. Итак, в работе рассмотрен общий случай линейного замкнутого оператора $A$. Полученные результаты, как видится, близки к предельным. Однако если $A$ порождает $C_{0}$-полугруппу, то теория единственности для обратной задачи $(1),(2)$ допускает дальнейшее развитие. Как выяснилось в процессе исследований, для такого оператора $A$ достаточно считать, что функция $\varphi(t)$ не обращается тождественно в нуль ни в какой окрестности точки $t=T$, т.е. что вьполняется только второе соотношение невырожденности (7). Тогда единственность решения обратной задачи $(1),(2)$ полностью выражается предположением $(\Lambda)$. Изложение результатов этого цикла готовится авторами к печати. Предварительное утверждение для дифференцируемых полугрупп см. в [8].

Отметим, что в статье совершенно не затрагивается тема разрешимости обратной задачи $(1),(2)$ с произвольными элементами $u_{0}, u_{1} \in D(A)$. Сошлемся здесь на работы [7], [9], где имеется теория для $C_{0}$-полугруппового случая.

Авторы признательны А. И. Прилепко, А. М. Седлецкому и М. Л. Содину за интерес, проявленньй к нашей работе, и А. Б. Костину за плодотворные обсуждения в процессе исследований. 


\section{СПИСОК ЦИТИРОВАННОЙ ЛИТЕРАТУРЫ}

[1] Prilepko A. I., Orlovsky D. G., Vasin I. A. Methods for Solving Inverse Problems in Mathematical Physics. New York-Basel: Marcel Dekker, 2000.

[2] Искендеров А. Д. Некоторые обратные задачи об определении правых частей дифференциальных уравнений // Изв. АН Азерб. ССР. Сер. физ. -техн. и матем. наук. 1976. № 2. C. $58-63$.

[3] Егоров А. И. Оптимальное управление тепловыми и диффузионными процессами. М.: Наука, 1978.

[4] Орловский Д. Г. К задаче определения параметра эволюционного уравнения // Дифференц. уравнения. 1990. Т. 26. № 9. С. 1614-1621.

[5] Rundell W. Determination of an unknown non-homogeneous term in a linear partial differential equation from overspecified boundary data // Appl. Anal. 1980. V. 10. № 3. P. 231-242.

[6] Эйдельман Ю.С. Единственность решения обратной задачи для дифференциального уравнения в банаховом пространстве // Дифференц. уравнения. 1987. Т. 23. № 9. С. 1647-1649.

[7] Эйдельман Ю. С. Одна обратная задача для эволюционного уравнения // Матем. заметки. 1991. Т. 49. № 5. С. 135-141.

[8] Eidelman Y.S. Conditions of existence and uniqueness of solution of inverse problem for the evolutionary equation // Докл. АН Украины. 1992. № 8. Р. 8-11.

[9] Тихонов И. В., Эйдельман Ю. С. Вопросы корректности прямых и обратных задач для эволюционного уравнения специального вида // Матем. заметки. 1994. Т. 56. № 2. С. 99-113.

[10] Аниконов Ю. Е., Вишневский М. П. Формулы в обратной задаче для эволюционного уравнения // Сиб. матем. ж. 1996. Т. 37. № 5. С. 963-976.

[11] Аюпова Н. Б., Голубятников В. П. О формальных решениях многомерных эволюционных уравнений // Матем. труды. Т. 1. № 2. Новосибирск: ИМ СО РАН, 1998. С. 3-23.

[12] Тихонов И. В., Эйдельман Ю. С. Единственность решения двухточечной обратной задачи для абстрактного дифференциального уравнения с неизвестньм параметром // Дифференц. уравнения. 2000. Т. 36. № 8. С. 1132-1133.

[13] Тихонов И. В., Эйдельман Ю. С. Обратная задача для дифференциального уравнения в банаховом пространстве и распределение нулей целой функции типа Миттаг-Лефффлера // Дифференц. уравнения. 2002. Т. 38. № 5. С. 637-644.

[14] Тихонов И. В. Соображения монотонности в обратной задаче для абстрактного дифференциального уравнения // Интегральные преобразования и спец. функции. Информ. бюлл. 2001. T. 2. №1. C. 119-128.

[15] Леонтьев А. Ф. Ряды экспонент. М.: Наука, 1976.

[16] Хилле Э., Филлипс Р. Функциональный анализ и полугруппы. М.: ИЛ, 1962.

[17] Левин Б.Я. Распределение корней целых функций. М.: ГИТТЛ, 1956.

[18] Любич Ю.И. О собственных и присоединенных функциях оператора дифференцирования // Изв. вузов. Матем. 1959. № 4. С. 94-103.

[19] Titchmarsh E. C. The zeros of certain integral functions // Proc. London Math. Soc. 1926. V. 25. № 4. P. 283-302.

[20] Федорюк М. В. Метод перевала. М.: Наука, 1977.

[21] Джрбашян М. М. Интегральные преобразования и представления функций в комплексной области. М.: Наука, 1966.

[22] Хёрмандер Л. Анализ линейных дифференциальных операторов с частньми производными Т. 1. Теория распределений и анализ Фурье. М.: Мир, 1986.

[23] Tychonoff A. Théorèmes d'unicité pour l'équation de la chaleur // Матем. сб. 1935. V. 42. № 2. P. 199-216.

[24] Мидзохота С. Теория уравнений с частньми производньми. М.: Мир, 1977.

[25] Любич Ю. И., Ткаченко В. А. Теория и некоторые применения локального преобразования Лапласа // Матем. сб. 1966. Т. 70. № 3. С. 416-437.

(И.В. Тихонов) Московский инженерно-физический институт

(Ю. С. Эйдельман) Университет г. Тель-Авив, Израиль

Поступило

E-mail: tikhonov@east.ru, eideyu@math.tau.ac.il

19.09.2002 